\title{
La percepción del profesorado sobre la enseñanza del deporte en la enseñanza secundaria obligatoria
}

Teachers' perception on teaching games in Secondary Education

Manuel Jesús López-Parralo

Manuel Tomás Abad-Robles ${ }^{2}$ (i)

Estefanía Castillo-Viera ${ }^{2}$ (1)

Francisco Javier Giménez-Fuentes-Guerra*²0

1. I.E.S. Diego Rodríguez Estrada (S. Juan del Puerto, Huelva), España.

2. Facultad de Educación, Psicología y Ciencias del Deporte. Universidad de Huelva, España.

\begin{abstract}
Resumen
Los profesores de Educación Física muestran una preocupación constante por hacer de su asignatura una materia con gran potencial educativo en la formación integral de los jóvenes. Dentro de esta asignatura, el deporte ocupa un lugar muy importante en las programaciones, a la vez que es también el que más controversias genera desde el punto de vista pedagógico. Estos dos aspectos son los motivos principales que nos llevan a estudiar cómo se aborda el proceso de enseñanza de este contenido durante la Enseñanza Secundaria Obligatoria (E.S.O.). Por ello, los objetivos principales de este estudio han sido: analizar la percepción del profesorado de educación física sobre el deporte como contenido de la ESO; y, en segundo lugar, describir posibles implicaciones didácticas que puedan ayudar a mejorar el proceso de enseñanza y aprendizaje de este contenido durante la E.S.O. Dentro del paradigma de investigación cualitativo, el diseño del estudio es fenomenológico, utilizando la entrevista semiestructurada como instrumento de investigación. Dicha entrevista fue aplicada en un grupo focal compuesto por 9 profesores: 8 hombres y 1 mujer; todos Graduados en Ciencias del Deporte; con una edad comprendida entre 28 y 32 años; y entre 4 y 6 años de experiencia docente. Como resultados más destacados, se puede indicar que los objetivos didácticos principales planteados por los profesores eran de carácter actitudinal y también de motivación del alumnado. Además, la orientación del deporte era de iniciación y promoción; los contenidos más desarrollados eran los técnico-tácticos y reglamentarios; y se aboga por una metodología activa en la que se incluyan valores como la colaboración o el respeto. En la evaluación destaca lo actitudinal sobre lo procedimental.
\end{abstract}

Palabras clave: Enseñanza del Deporte, Valores, Educación Física.

\begin{abstract}
Physical Education teachers show a constant concern for making their subject a subject with great educational potential in the integral formation of young people. Within this subject, sport occupies a very important place in the programming, while it is also the one that generates most controversy from the pedagogical point of view. These two aspects are the main reasons that lead us to study how the teaching process of this content is approached during Compulsory Secondary Education (C.S.E.). Therefore, the main objectives of this study have been: to analyze the perception of physical education teachers about sport as a content of C.S.E.; and, secondly, to describe possible didactic implications that may help to improve the teaching and learning process of this content during C.S.E. Within the qualitative research paradigm, the design of the study is phenomenological, using the semi-structured interview as a research instrument. These interviews were applied in a focus group. The most outstanding results are that the main teaching objectives set by the teachers were attitudinal and also motivational. In addition, the orientation of the sport was one of initiation and promotion; the most developed contents were the technical-tactical and regulatory ones; and an active methodology was advocated in which values such as collaboration or respect were included. In the evaluation, the attitudinal aspects are highlighted.
\end{abstract}

Keywords: Teaching Games, Values, Physical Education.

* Autor de correspondencia: Fco. Javier Giménez Fuentes-Guerra, jfuentes@uhu.es

Recibido: 10 de mayo de 2020

Aceptado: 27 de junio de 2020

Publicado: 14 de julio de 2020

Como citar (APA): López-Parralo, M. J., Abad-Robles, M. T., Castillo-Viera, E., \& Giménez-Fuentes-Guerra, F. J. (2020). La percepción del profesorado sobre la enseñanza del deporte en la enseñanza secundaria obligatoria. JUMP, (2), 57-67. https://doi.org/10.17561/jump. n2.6

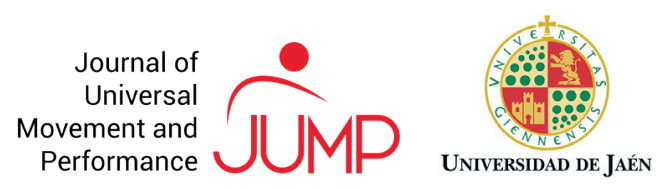




\section{Introducción}

Existe una constante preocupación por parte del profesorado Educación Física (EF) por hacer de esta asignatura, y por tanto, de todos los contenidos que en ella se imparten, una materia con gran potencial educativo en la formación integral de los jóvenes (Devís y Peiró, 2011; Feu, 2002; Fraile, 1996; García, 1990; Méndez y Fernández, 2011; Renzi, 2009). Dentro de ésta, el deporte ocupa un lugar muy importante en las programaciones como confirma el estudio de Robles, Giménez y Abad (2010), donde se pone de manifiesto el peso que este contenido tiene con respecto a los otros bloques de la Educación Física en la E.S.O., siendo el más trabajado por el profesorado tanto en número de sesiones como de unidades didácticas. Pero esta relevancia, no ha estado exenta de diversas críticas cuando éste no ha sido bien planteado, porque como afirma Calzada (2004, p. 44) "en general la práctica deportiva corresponde a planteamientos competitivos, selectivos, y restringidos a una sola especialidad, que no siempre son compatibles con las intenciones educativas del currículo escolar".

Debido a la importancia del deporte y a planteamientos inadecuados por una parte del profesorado, este contenido empieza a ser estudiado buscando alternativas más validas desde una perspectiva mucho más formativa. En este sentido, en torno a los años ochenta del siglo XX se plantea la existencia de dos enfoques metodológicos predominantes en la enseñanza deportiva: un enfoque más tradicional y técnico, frente a otro enfoque más activo y centrado en la táctica (Almond, 1986; Devís y Sánchez, 1996; Méndez, 1999; Castejón, 2003). Águila y Casimiro (2001) afirman que los métodos tradicionales son más típicos del entrenamiento deportivo, donde los entrenadores carecen de la formación pedagógica y didáctica y, por tanto, basan sus planteamientos de aprendizaje en la consecución de un abanico más o menos amplio de elementos técnicos individuales. Estas críticas, junto a las aportaciones de autores como Espada, Clemente, Santacruz y Gallardo (2013) que afirman que todavía se pueden encontrar demasiados trabajos con planteamientos tradicionales con poca valía educativa, hacen que haya que seguir siendo críticos y buscando nuevos planteamientos. De esta manera, cada vez son más comunes las opiniones que plantean que la enseñanza del deporte debe evolucionar hacia modelos más comprensivos y educativos (Castejón, Giménez, Jiménez y López, 2013; Valero, 2006). Además, coincidiendo con Giménez y Rodríguez (2006), esta necesaria evolución debe tener más en cuenta al niño, sus características y el contexto donde se mueve, y darle menos importancia al desarrollo tradicional de la técnica en beneficio de la comprensión y la participación de todos los alumnos independientemente de su nivel de aptitud.

Para seguir justificando la necesidad de nuevos planteamientos didácticos de enseñanza del deporte, resulta necesario destacar numerosas aportaciones de autores como Giménez (2003), Gutiérrez (1995) u Ortega et al. (2012, 2015), que enfatizan en las condiciones en las que se realice dicha práctica. En definitiva, y coincidiendo con autores como González, García, Contreras y Sánchez-Mora (2009), se puede afirmar que el deporte debe enseñarse en la E.S.O. a través de modelos activos donde el proceso de enseñanza y aprendizaje se adapte a las características del alumnado, siendo éstos los verdaderos protagonistas. Todo ello bajo un prisma formativo, donde, además de los elementos específicos del deporte, se utilice el mismo como medio para una formación más integral.

Estos planteamientos, además de un cambio didáctico, suponen también un cambio pedagógico que se puede observar claramente en la necesidad de promoción de los valores tan importantes en esta etapa educativa. Para Lee, Whitehead y Balchin (2000), existe un auge en Europa por el estudio de las relaciones entre deporte y transmisión de valores. Álamo, Amador y Pintor (2002, p.1), justifican la presencia de este contenido en la escuela afirmando que "el deporte escolar, aparece por lo general asociado a valores y actitudes como la cooperación, el diálogo, el respeto, la responsabilidad, la sinceridad o la creatividad. Este es argumento suficiente para que la práctica deportiva goce de presencia destacable en muchas de las actividades que se desarrollan con el objetivo de contribuir al proceso educativo de los jóvenes". Del mismo modo, Grenn y Gabbard (1999) hablan de la importancia de la transmisión de valores a través del deporte, incidiendo en educar a los jóvenes en la "deportividad". Arnold (2001) añade que la enseñanza del deporte bien orientado es en sí una forma de educación moral. Por todo ello, se puede considerar importante 
incluir este contenido como actividad curricular, pero a la vez se piensa que se debe investigar el proceso para conocer la realidad, ser crítico con ella, y poder plantear alternativas educativas integrales.

En definitiva, el potencial de la educación física y la necesidad de hacer del deporte un contenido verdaderamente educativo, son los motivos principales que justifican el estudio. Por ello, los objetivos principales del mismo han sido: Analizar la percepción del profesorado de educación física sobre el deporte como contenido de la ESO; y, en segundo lugar, describir posibles implicaciones didácticas que puedan ayudar a mejorar el proceso de enseñanza y aprendizaje de este contenido durante la E.S.O.

\section{Materiales y método}

\section{Diseño e Instrumentos}

Dentro del paradigma de investigación cualitativo, el diseño del estudio es fenomenológico (Hernández, Fernández y Batista, 2006) ya que es la forma más adecuada para entender a cada una de las personas que aporta la información, sus motivos, ideas y conocimientos. Su propio punto de vista permite obtener una información más precisa. Además, el intercambio de información entre iguales, abonado por un interlocutor como es el investigador externo con el fin de que se contrasten entre ellos, permite disentir o estar de acuerdo entre ellos y facilita la obtención de cambios en su forma de proceder, tanto en el diseño como en la puesta en práctica de la enseñanza del deporte y sobre las repercusiones que tiene para su trabajo.

Como instrumento de investigación se ha utilizado la entrevista semiestructurada (Cohen y Manion, 1990; Palacios, 2000). Dicha entrevista se llevó a cabo al principio con la idea de conocer el punto de partida de los profesores sobre los temas a desarrollar en el grupo de trabajo; y al final para conocer la incidencia de la formación en dichos profesores.

De cara a la validación del instrumento, se diseña el primer borrador de la entrevista, y éste se pasa a un grupo de 5 expertos que contaban con el siguiente perfil: profesores doctores universitarios, más de 15 años de experiencia en investigación cualitativa en CC del Deporte, y en todos los casos con publicaciones de calidad relacionadas con el objeto de estudio. Para la validación de la misma, cada experto ha utilizado una escala tipo Likert, valorando cada pregunta de 1 a 4 (donde 1 se corresponde con "en desacuerdo" y 4 con "totalmente de acuerdo"). Esta escala se aplica al contenido, la importancia y la adecuación de cada pregunta para el desarrollo del presente estudio. Además, se les brindaba la oportunidad de realizar cualquier sugerencia a las preguntas, aportando cuestiones alternativas a las ya elaboradas o añadiendo cualquier otra que considerara oportuna.

\section{Participantes}

Los participantes iniciales fueron 10 profesores de E.F. que impartían su docencia en la E.S.O. Uno de los participantes abandonó al inicio, por lo que la muestra final fue de 9 profesores; 8 hombres y 1 mujer; todos Graduados en Ciencias del Deporte; con una edad comprendida entre 28 y 32 años; y entre 4 y 6 años de experiencia docente.

\section{Procedimiento}

De forma voluntaria se ofreció, desde el Centro del Profesorado de Huelva a principios de curso, a todos los docentes de Educación Física de E.S.O., la posibilidad de participar en el Grupo de Trabajo denominado "El deporte en la E.S.O. Análisis y perspectivas educativas de actuación". La duración del grupo de trabajo fue de un curso académico completo (2017-18). En el mismo se llevaron a cabo 18 sesiones con una duración de dos horas y media cada una. Además, se diseñaron tres unidades didácticas para desarrollar a lo largo del curso (un deporte psicomotriz: el atletismo; uno de oposición: judo; y uno de colaboración-oposición: baloncesto).

El objetivo específico de dicho grupo fue la reflexión, intercambio de experiencias, y debate sobre cómo hay que trabajar el deporte en la E.S.O., Ilevándolo siempre a la práctica. También, un objetivo importante del mismo consistió en la adquisición de hábitos de trabajo en grupo y de investigación sobre la práctica.

Todo ello se desarrolló a través del diseño de Unidades Didácticas en grupo; la puesta en práctica de las mismas; la reflexión a través del diario del profesor; y el intercambio de reflexiones recogidas en los diarios. Estas fases se corresponden con 
el proceso de reflexión colaborativa en espiral propuestas por Kemmis y McTgagart (1982): planificación, acción, observación y reflexión.

\section{Análisis de los datos}

El análisis de los datos de este trabajo se centra exclusivamente en las entrevistas realizadas. Se han seguido las recomendaciones de Taylor y Bogdan (1990) con el fin de poder presentar unos resultados coherentes yútiles para la investigación: descubrimiento, codificación y relativización. Las entrevistas, inicial y final, son grabadas y transcritas en un procesador de textos. A continuación, se establecen los códigos que servirán para agrupar las ideas que subyacen en las respuestas de los profesores participantes.

Una vez seleccionados los códigos, lo siguiente fue asignarlos a los textos. Para ello, siguiendo las recomendaciones de Rodríguez (1994), se llevó a cabo por varios investigadores (tres en este caso). Se realizaron varias reuniones conjuntas de codificación con el objetivo de alcanzar el máximo consenso posible, llegando en la última a un grado de fiabilidad interna entre codificadores del 94\%. No obstante, a fin de paliar los acuerdos debidos exclusivamente al azar, se calculó el Índice de Kappa (Cohen, 1960) de las tres reuniones con un nivel de significación de 0.842 utilizando el programa informático SPSS 21.0 (Statistical Program of Social Sciences).

Por último, una vez transcritas y codificadas las entrevistas, se introdujeron los códigos en el programa informático MXQDA 2018 que facilitó el proceso de reducir los datos, contar los códigos y manejar los textos para la elaboración del informe de investigación.

\section{Análisis y discusión de los resultados}

Se analizan, a continuación, los resultados obtenidos de las entrevistas realizadas al inicio y al finalizar el grupo de trabajo. Para realizar dicho análisis y discusión de los resultados, en primer lugar, se comienza por los objetivos que los profesores se marcaban en la enseñanza de este contenido. Además, plantean la orientación que debía tener y los principales contenidos que se trabajaban. A partir de estas consideraciones iniciales, se analizan los aspectos metodológicos y técnico-tácticos; y se termina estudiando los valores del deporte que se fomentan y cómo se puede evaluar. De esta forma los resultados obtenidos pueden ser mucho más comprensibles para el lector.

En total se analizaron un total de 868 extractos de texto, con los porcentajes que se detallan a continuación. Como sepuedeobservar claramente, los códigos "metodología" y elementos "técnicotácticos" son los más presentes (51,95\% del total entre los dos); mientras que los menos numerosos fueron "objetivos", "orientación del deporte" y "valores" con menos del $9 \%$. En una situación intermedia, con un 10'94\%, se sitúan los códigos "contenidos deportivos" y "evaluación".

Tabla 2. Códigos, frecuencias y porcentajes del análisis de las entrevistas realizadas.

\begin{tabular}{lll}
\hline CÓDIGOS & Frecuencia & Porcentaje \\
\hline Objetivos & 75 & 8,64 \\
Orientación del deporte & 75 & 8,64 \\
Contenidos deportivos & 95 & 10,94 \\
Metodología & 252 & 29,03 \\
Elem. técnico-tácticos & 199 & 22,92 \\
Valores & 77 & 8,87 \\
Evaluación & 95 & 10,94 \\
\hline Total & 868 & $100 \%$ \\
\hline
\end{tabular}

Tabla 1. Dimensiones y códigos de la entrevista semiestructurada.

\begin{tabular}{|c|c|c|}
\hline DIMENSIÓN & CÓDIGOS & DESCRIPCIÓN DE LOS CÓDIGOS \\
\hline \multirow{7}{*}{ Deporte en la ESO } & Objetivos & Finalidades que se persiguen con la práctica deportiva \\
\hline & $\begin{array}{l}\text { Orientación del deporte en la } \\
\text { E.S.O. }\end{array}$ & Tipo de orientación que se le da al deporte: recreación, competición, etc. \\
\hline & Contenidos deportivos & $\begin{array}{l}\text { Tipo de contenidos que se desarrollan: técnicos, tácticos, físicos, psicológicos, } \\
\text { etc. }\end{array}$ \\
\hline & Metodología enseñando deporte & Intervención didáctica que se emplea por parte del profesor \\
\hline & Elementos técnico-tácticos & Aspectos técnico y tácticos que se desarrollan \\
\hline & Valores & Valores educativos que se fomentan \\
\hline & Evaluación en el deporte & Cómo se evalúa el contenido \\
\hline
\end{tabular}




\section{Objetivos}

En relación con los principales objetivos que el profesorado se plantea cuando imparte el deporte en sus clases de Educación Física, todos dieron especial relevancia a los objetivos actitudinales tanto en las entrevistas iniciales como en las finales. Estos datos coinciden con los de otros estudios (Gaviria y Castejón, 2013; Giménez y Rodríguez, 2006).

"...el objetivo principal en el instituto, en el tema de los deportes, es que se relacionen, que sepan jugar, que sepan ganar, que sepan perder, que no les importe jugar con las niñas o con los niños, que sean capaces de hacer una jornada con algún instituto de la provincia. El objetivo principal es que sepan relacionarse, que sepan respetarse los unos a los otros" (Profesor 4, entrevista inicial).

"...fundamentalmente, que tengan una actitud positiva hacia el deporte, que, como está muy de moda lo de los valores, se socialicen, que sepan respetar las decisiones de sus compañeros..." (Profesor 7, entrevista inicial).

"Cuando enseño deporte en la E.S.O. me planteo lo mismo que cuando enseño expresión corporal o cuando enseño juegos y actividades de la naturaleza, el primer objetivo que yo intento con los alumnos, y además se lo recalco bien, es lograr una actitud favorable en lo que es el proceso de enseñanza y aprendizaje; ¿y actitud favorable en qué sentido?, pues en la participación, en la motivación, en el interés y en la cooperación que muestre de cara al aprendizaje de esa actividad físicodeportiva. Ese es el primer objetivo que yo marco..." (Profesor 2, entrevista final).

También destacó que entre los objetivos que se planteaban cuando enseñaban deporte en la E.S.O., la diversión apareció como objetivo prioritario en bastantes casos (Giménez, 2003).

"El primer objetivo que me planteo es que se diviertan..." (Profesor 2, entrevista inicial).

"Ante todo, que los chiquillos disfruten y se lo pasen bien, eso lo primero, y después, la formación física" (Profesor 3, entrevista inicial).

"...mi objetivo no es que sepa jugarlo bien para que sea útil en sus competiciones y en los entrenamientos. No. Simplemente, que se diviertan y si oyes que tres tardes en semana se van a jugar a algo nuevo, que yo les he enseñado, pues estupendo; y a parte también que aprendan, que se formen como personas. Le doy mucha importancia a que ellos a través del deporte adquieran valores, no sólo los de compañerismo, cooperación, sino también que tengan unas metas a conseguir" (Profesor 7, entrevista final).

Por otro lado, Thomas, Lee y Thomas (2003) y Silverman (2005) consideran que en la enseñanza del deporte en Educación Física hay que mejorar la condición física y la salud de los niños. En este sentido, cabe destacar que los objetivos saludables también formaron parte de las prioridades de algunos de los profesores de la investigación, y la utilización del deporte como herramienta de salud.

"Para fomentar los hábitos de salud de los niños..." (Profesor 1, entrevista inicial).

"Luego, también meto que adquieran unos hábitos higiénicos como que se aseen y cambien las camisetas" (Profesor 7, entrevista inicial).

En relación con la salud y la necesidad de fomentar hábitos, también apareció como objetivo, aunque en menor medida, que el alumnado aprendiera elementos que puedan utilizar en su tiempo libre, lo cual coincide con el trabajo de Ramos, Valdemoro, Sanz y Ponce de León (2007), en el que queda claro la influencia que el profesorado puede tener en este sentido.

"Porque es un medio, una herramienta para hacer actividad física después. Un niño no se va a correr por las tardes, pero sí hace deporte que es algo que le gusta" (Profesor 7, entrevista inicial).

"Sí, que se muevan, y luego, pues les digo: oye que ha empezado la escuela deportiva de fútbol, de tenis. Si soy capaz de trasladar lo que hacemos en el instituto a por las 
tardes, bien con las clases, bien con los campeonatos del recreo y con lo que hagan por la tarde, pues ya sería un éxito" (Profesor 4 , entrevista final).

"A parte de que el niño conozca el deporte de una manera básica, fundamentalmente es que aplique ese deporte y que intente divertirse, que él por sí sólo pueda ocupar su tiempo libre practicando ese deporte" (Profesor 7, entrevista final).

Por último, hay que destacar la interesante aportación de uno de los miembros del grupo de trabajo en la entrevista final, que incidió en que sus objetivos están condicionados por el contexto donde enseñan.

"Va en función de las necesidades de los alumnos. Yo el año pasado daba mucha importancia a los valores, por las necesidades de mis alumnos y, sin embargo, este año tengo otros alumnos que no les encuentro esas necesidades." (Profesor 7 , entrevista final).

\section{Orientación del deporte en la E.S.O.}

La mayoría de los profesores investigados pensaban que la orientación más adecuada a la hora de enseñar deporte en la E.S.O. era la iniciación y la promoción deportiva. Esto se observa tanto en las entrevistas iniciales como en las finales. En este mismo sentido se pronuncian Fraile (1996) y Giménez (2003). En concreto, en las entrevistas iniciales, uno de los sujetos justificó la consideración anterior por el nivel que tenían la mayoría de alumnos/as de la E.S.O. En esta línea, sugería que la orientación fuese de iniciación y no de perfeccionamiento.

"Yo veo que, si consiguiéramos que los niños se iniciaran deportivamente en unas especialidades deportivas variadas, no sé si dos, tres, cuatro, cinco las que sea, con eso me conformaría yo" (Profesor 2, entrevista inicial).

"Yo creo que profundizar en la E.S.O. no tiene sentido profundizar, yo creo que es un error enseñar a los niños..., para mí, fundamental, esa es la orientación, iniciar a los niños en el deporte, porque con dos horas a la semana tampoco hay para más" (Profesor 4, entrevista inicial).

"Yo creo que habría que dar iniciación deportiva, y los niños, una vez que conozcan esos deportes, piensen si quieren seguir profundizando, pero a nivel individual, porque en las clases que tenemos no nos da tiempo profundizar en ningún deporte" (Profesor 2 , entrevista final).

"Yo creo que esa es la orientación, porque, por ejemplo, sacar a delante las clases de baloncesto, no con el grupo del diario, sino con otro grupo, me costó la misma vida, porque, cuando terminábamos las clases los nenes, estaban deseando que terminaran, y los otros se iban con ganas de que viniese la siguiente clase. Si tú aburres a los nenes un día, otro día y otro día, te van a venir a educación física igual que van a matemáticas. Yo creo que se tienen que ir con ilusión de la clase de educación física. Yo creo que sí, que esa es la orientación que hay que darle" (Profesor 4, entrevista final).

Además, de acuerdo con Ginsburg et al. (2007) en sus opiniones sobre adaptar la enseñanza deportiva a los niveles del alumnado, uno de los miembros justificó esta orientación a la iniciación deportiva con el hecho de que es el nivel que tiene la mayoría de los alumnos de la E.S.O.

"... porque tenemos veinte o treinta alumnos y no podemos hacer tantos niveles distintos, tú tienes que dar una clase de un grupo de chavales y te tienes que amoldar a la media, y la media es que no tienen ni idea, es que no hacen actividades deportivas por la tarde; después hay uno o dos que son muy buenos, pero yo particularmente no hago una clase para esos dos, se tendrán que acoplar". (Profesor 7, entrevista inicial).

Por otro lado, dos de los sujetos entrevistados, plantearon en las entrevistas iniciales que en segundo ciclo de la E.S.O. habría que profundizar un poco más. Sin embargo, uno de ellos cambió de opinión después del desarrollo del seminario de formación, optando por una enseñanza del deporte en la E.S.O. basada en la iniciación deportiva. 
"Hombre, yo creo que en primero y segundo de E.S.O., que es donde yo doy creo que hay que dar iniciación, después en tercero y cuarto y bachillerato hay que profundizar un poquito más, sin llegar a agobiar a los niños" (Profesor 1, entrevista inicial).

"Podemos establecer para el primer ciclo aspectos generales de varios deportes y después en tercero y cuarto profundizar un poco más" (Profesor 5, entrevista inicial).

"Hay que fomentar la iniciación a muchos deportes, que les atraigan diferentes prácticas deportivas" (Profesor 5, entrevista final).

\section{Contenidos deportivos}

Sobre qué enseñan los profesores de nuestro estudio cuando tratan los contenidos deportivos en la E.S.O., cabe señalar que, tanto en las entrevistas iniciales como en las finales, la mayoría respondió que los contenidos que enseñaban eran fundamentalmente los elementos técnico-tácticos y el reglamento de cada deporte.

"Trabajo elementos técnico-tácticos, pero de una forma muy general... Las reglas muy poco, lo básico. Por ejemplo, en voleibol la puntuación, las rotaciones" (Profesor 3, entrevista inicial).

"Por ejemplo, si yo digo voy a trabajar el pase, pues digo: jugamos dos contra dos y no vale botar. Entonces, el jugador sabe que, si da dos pasos con el balón en las manos, se le pita pasos, y así estoy trabajando el aspecto reglamentario. Pero mi objetivo fundamental son los elementos técnicotácticos" (Profesor 5, entrevista inicial).

Por otro lado, hay que destacar, que a diferencia de lo que ocurría en la categoría anterior, donde todos daban mucha importancia a los objetivos actitudinales, aquí sólo un miembro del grupo de trabajo incluyó, en la entrevista inicial, las actitudes como contenido importante a desarrollar (Gaviria y Castejón, 2013).

"...la actitud es sencilla, hay unas reglas, vengo a mi hora, traigo mi ropa, respeto a mi compañero, luego al profesor y respeto al material. Y a partir de ahí, empezamos a funcionar..." (Profesor 4, entrevista inicial).

También apareció dentro del análisis de este código, por parte de dos profesores y sólo en la entrevista final, la utilización del deporte como medio para desarrollar otros contenidos de educación física (Robles, Giménez y Abad, 2010; Ureña, Alarcón y Ureña, 2009).

"Lo veo importante porque es un medio en el que se recoge muchos aspectos a la hora de enseñar en clase, yo que sé, a la hora de la sociabilidad de los niños, a la hora de poder enfocar, de trabajar distintos aspectos de la educación física, a la hora de trabajarlos. En vez de trabajarlos de forma específica, lo puedes trabajar, las cualidades físicas, las puedes trabajar, a través de un deporte, entonces es más fácil porque los niños disfrutando, están intentando conseguir unos objetivos, aprendiendo una determinada cosa y a la vez están trabajando las distintas cualidades físicas que se debe trabajar en la materia" (Profesor 3, entrevista final).

"...el deporte lo utilizo yo para enseñar casi todo, es un medio para enseñar casi todos los contenidos que yo doy en las clases, desde la resistencia, la fuerza, la velocidad, todo eso lo hago en forma de juego o en forma de deporte" (Profesor 5, entrevista final).

\section{Elementos técnico-tácticos}

Este apartado hace referencia a los comentarios que aparecieron referidos a la técnica o táctica en general, o cuando aludían a elementos técnicotácticos concretos. Así, se halló que en las entrevistas iniciales algunos sujetos decían que se centraban en la técnica cuando enseñaban deporte en línea con planteamientos más tradicionales definidos por Alarcón et al. (2010).

"...más la técnica que la táctica, la táctica no creo que sea importante para estos niveles, para unos niños tan pequeños" (Profesor 1, entrevista inicial) 
"...hombre..., la técnica en un deporte tiene importancia a nivel individual" (Profesor 2 , entrevista inicial).

"A la técnica, porque para dar una serie de cosas si no aprenden bien la técnica no lo van a poder hacer. A la hora de trabajar la táctica tienes que trabajar antes la técnica" (Profesor 3, entrevista inicial).

Estos comentarios cambiaron una vez que se desarrolló el seminario de formación, ya que en las entrevistas finales la mayoría contestaron que estos elementos los enseñaban de manera conjunta y no aislada. Además, uno de ellos, coincidió con los planteamientos de Méndez (1999), aludiendo a que cuando enseña deporte lo que trata es de desarrollar el pensamiento táctico del alumnado.

"Siempre a través del juego. Pues puedo hacer juegos enseñando tanto la técnica como la táctica, todo junto" (Profesor 1, entrevista final).

"Yo cuando me planteo que el niño desarrolle su pensamiento táctico, es decir, no me planteo que adquiera elementos tácticos para la práctica de ese deporte, sino que tenga buena transferencia para la práctica de otro deporte colectivo" (Sujeto 7, entrevista final).

\section{Metodología enseñando deporte}

En el análisis de este código se observó, en línea con el código anterior, cómo algunos profesores incidían, sobre todo, en aspectos técnicos más tradicionales antes de realizar la formación en grupo.

"Pues, suelo poner a los niños delante mía, les explico los gestos que vaya a dar, explico ese gesto, lo vamos practicando las veces que haga falta, después cambiamos de gesto, le explico el ejercicio y ellos lo practican" (Profesor 1, entrevista inicial).

"Por ejemplo, un relevo con lanzamiento de balón, carrera de relevo con lanzamiento de balón. Entonces, el relevo va a consistir en llegar a un punto, coger el balón, no tiene por qué ser el balón de baloncesto, puede ser cualquier otro balón, lanzarlo en la forma que yo he explicado de forma técnica que había que lanzarlo..." (Profesor 2, entrevista inicial).

Al terminar el grupo de trabajo la evolución es positiva y se pueden ver algunos comentarios interesantes en este sentido, en línea con las propuestas activas de Castejón et al. (2013) en las que el planteamiento de la enseñanza es más global, lúdico y comprensiva, se evoluciona de lo táctico a los técnicos, se adapta el material y las reglas, etc.

"Antes lo solía hacer más analítico y con menos juegos" (Profesor 1, entrevista final).

"...los 10 pases, todo el mundo sabe cómo es, y tanto con balones de baloncesto, como con balones de voleibol, o con balones de fútbol, incluso con los pies..." (Profesor 2, entrevista final).

"Antes era más analítico, más técnico, intentaba más que aprendieran gestos técnicos; le he dado una vuelta y quizás lo haga todo con juegos, que ellos piensen jah! pues esto se parece a..." (Profesor 6, entrevista final).

De todas formas, también se encuentran aportaciones positivas de algunos profesores desde el inicio del grupo de trabajo, dando ejemplos coherentes con el tipo de metodología que utilizaban enseñando deporte en la E.S.O. Esta metodología es fundamentalmente activa, basada en situaciones reales de juego, siempre con oposición, donde el alumnado debe buscar soluciones a los problemas motrices que se le plantean. Esta metodología coincide con la propuesta para la enseñanza del deporte en la escuela de autores como Castejón et al. (2003, 2013), Giménez (2003), o Méndez (1999).

\section{"Juegos, sobre todo, juegos con reglas de provocación donde tengan que practicar el objetivo que tengan que conseguir" (Profesor 5, entrevista inicial).}

"Por ejemplo, si yo digo..., voy a trabajar el pase, pues digo, jugamos dos contra dos y no vale botar..." (Profesor 5, entrevista inicial). 


\section{Valores}

La educación en valores fue valorada como muy necesaria por parte de todos los profesores participantes. En línea con el estudio de Gaviria y Castejón (2013), los valores sobre los que más insistieron fueron la cooperación, el respeto y la salud.

"Trabajo en equipo, que los niños respeten sus propias limitaciones y la de sus propios compañeros, el respeto, la cooperación..." (Profesor 1, entrevista inicial).

"...que sepan ganar, que sepan perder..." (Profesor 4, entrevista inicial).

"...comportamientos con sus compañeros, la relación con sus compañeros..." (Profesor 1, entrevista final).

"La socialización, es una cosa que, a mí, una cosa que yo tengo mucho en cuenta. Que mis alumnos se relacionen unos con otros, porque muchas veces, yo veo que, en clase, sí, tienen un contacto, pero tienen un contacto con el que está al lado y con el otro del lado, y no. Entonces, yo el deporte lo veo como una materia muy buena para trabajar en grupo y alcanzar un determinado objetivo en sí..." (Profesor 3, entrevista final).

"...utilizar el deporte para transmitir valores como el de hábito de vida saludable, o valores de ayudar al compañero, cuidar el material..." (Profesor 4, entrevista final).

González (2005, p.3) plantea que "para hablar de una enseñanza deportiva educativa, se debe trascender del aprendizaje de lo meramente motriz para auspiciar otros ámbitos que tradicionalmente han sido soslayados como el cognitivo y, sobre todo, el moral". No cabe duda de que el deporte es una potente herramienta para la transmisión de valores en la E.S.O. Se considera fundamental la utilización de este contenido para formar ciudadanos con una escala de actitudes y valores adecuada. Además, se estima que uno de los objetivos principales en la enseñanza del deporte en la Enseñanza Secundaria es la transmisión de actitudes, valores y normas (Álamo et al, 2002; Fraile, 1996; Giménez, 2003; Grenn y Gabbard, 1999; Trepat, 1995).
Por último, coincidiendo con Jiménez (2006), en contextos desfavorecidos es más importante aún la transmisión de actitudes, valores y normas, y el deporte es un instrumento fundamental para conseguirlo.

"Sí, es un instituto que con los años ha cogido la fama de mala convivencia...; entonces utilizo el deporte como medio para educar, para mejorar la convivencia del centro" (Profesor 4, entrevista inicial).

"No, en primero la parte conceptual y procedimental muy poquito porque son chavales de una barriada marginal, donde la parte actitudinal es muy importante para ellos" (Profesor 7, entrevista inicial).

\section{Evaluación}

Aunque anteriormente en la categoría contenidos deportivos sólo un miembro hablaba que trabajaba como contenido las actitudes y valores en sus clases, ahora, cuando se habla de evaluación, la mayoría contestó que para calificar a sus alumnos el mayor peso de la nota recaía en las actitudes, lo cual coincide con lo manifestado por Pérez-Pueyo (2016).

"...yo creo que más que evaluar aspectos técnicos en los niños deberíamos echar más cuenta a las actitudes..." (Profesor 1, entrevista inicial).

"Pues mira, lo primero, la actitud, que le doy un porcentaje alto. Así, el niño ha participado y si ha estado motivado en las clases, ha ayudado con el material, si los equipos los ha formado mixtos y no ha protestado, etc., etc.; lo segundo los procedimientos..." (Profesor 2, entrevista inicial).

"A las actitudes, después procedimientos y después conceptos. Los porcentajes serían: actitud $70 \%, 20 \%$ procedimientos, y $10 \%$ conceptos" (Profesor 3, entrevista inicial).

"En primer lugar, a las actitudes, en segundo lugar, a los procedimientos y, en último lugar, a los conceptos" (Profesor 1, entrevista final). 
Sobre las técnicas e instrumentos que utilizan para evaluar, la mayoría realiza una evaluación más subjetiva.

"Para hacerlo objetivo habría que hacer exámenes, y perdería tres sesiones, habría que hacer test físicos y técnico-tácticos, y perdería otras clases. Además, yo no me fijo si el niño es capaz de hacer tantos toques. Al fin y al cabo, es todo subjetividad, lo que yo percibo" (Profesor 7, entrevista inicial).

Solamente un miembro utilizaba técnicas objetivas, pero las combinaba con subjetivas.

"Hojas de observación, donde evalúo los gestos técnicos y otras donde aparece lo que es la participación, el respeto..." (Profesor 3, entrevista inicial).

\section{Conclusiones}

En relación al primer objetivo del estudio: Analizar la percepción del profesorado de educación física sobre el deporte como contenido de la ESO; se puede destacar que los objetivos a los que el profesorado da más importancia en la enseñanza del deporte en la E.S.O. son de carácter actitudinal, aunque también tienen mucha presencia los objetivos lúdicos o motivadores. En menor medida, aparecen objetivos relacionados con la salud y la promoción de hábitos de práctica.

En cuanto a la orientación que debe tener el deporte en esta etapa educativa, los profesores indican que debe ser de iniciación y de promoción deportiva, evitando todo tipo de selección y el exceso de competitividad típica del deporte de competición extracurricular.

Para los entrevistados, los contenidos que se desarrollan en clase son principalmente técnicotácticos y reglamentarios. En algunos casos, el deporte también es utilizado como medio para desarrollar otros contenidos de educación física.

En relación con los contenidos técnico-tácticos y con la metodología, se produce una evolución positiva en el pensamiento del profesorado, ya que, al finalizar el grupo de trabajo, de forma unánime, todos se separan de paradigmas técnicos tradicionales, y abogan por una enseñanza activa y global donde el alumnado es el verdadero protagonista.
En clase de educación física, todos consideran esencial dotar al deporte de valores educativos que ayuden a sus alumnos a mejorar como personas. Entre los principales valores que plantea el profesorado, están la cooperación, el respeto y la salud. En este sentido, en estrecha relación con lo expresado anteriormente, el profesorado evalúa sobre todo los aspectos actitudinales. En menor medida evalúa aspectos procedimentales y conceptuales.

\section{Aplicaciones prácticas}

En relación al segundo objetivo planteado en este trabajo: Describir posibles implicaciones didácticas que puedan ayudar a mejorar el proceso de enseñanza y aprendizaje de este contenido durante la E.S.O., se podrían indicar las siguientes aplicaciones prácticas: dar especial importancia a las actitudes y a los valores en todo el proceso de aprendizaje de un deporte incluyendo las mismas en cada sesión de entrenamiento; evitar un exceso de competitividad que pudiera influirnegativamente en la motivación de los estudiantes con menores aptitudes; e implementar planteamientos metodológicos activos donde los chicos y chicas de Secundaria se sientan verdaderamente partícipes de su evolución y aprendizaje.

\section{Referencias}

Águila, C. \& Casimiro A. (2001). Iniciación a los deportes colectivos. En F. Ruiz; A. García; A. Casimiro. La iniciación deportiva basada en los deportes colectivos. Madrid: Gymnos.

Álamo, J. M., Amador, F. \& Pintor, P. (2002). Función social del deporte. El entrenador del deporte escolar. Lecturas Educación Física y Deportes, 45

Alarcón, F., Cárdenas, D., Miranda, M.T. \& Ureña, N. (2010). Metodología de enseñanza de los deportes de equipo. Revista de Investigación en Educación, 7, 91-103.

Almond, L. (1986). Reflecting on themes: a games classification. En R. Thorpe, D. Bunker \& L. Almond (Eds). Rethinking Games Teaching (pp.71-72). Loughborough University.

Arnold, P. J. (2001). Sport, moral development, and the role of the teacher. Implications for research and moral education. Quest 53, 135-150. https://doi.org/10.1080/00336297.2001.10491734

Calzada, A. (2004). Deporte y educación. Revista de Educación, 335, 45-60. http://www.revistaeducacion.educacion.es

Castejón, F. J. (2003). Iniciación deportiva. La enseñanza y el aprendizaje comprensivo en el deporte. Sevilla: Wanceulen SL.

Castejón, F.J., Giménez, F.J., Jiménez, F. \& López, V. (2013). Formación deportiva como eje central de la enseñanza del deporte. En F.J. Castejón, F.J. Giménez, F. Jiménez \& V. López (Eds). Formación deportiva (pp. 15-37). Sevilla: Wanceulen.

Cohen, J. (1960). A coefficient of agreement for nominals scales. Educational and Psychological measure-ment, 20, 37-46. https://doi.org/10.1177/001316446002000104 
Cohen, L. \& Manion, L. (1990). La entrevista. En Cohen, L. \& Manion, L. Métodos de investigación educativa. Madrid: La Muralla.

Devís, J. y Peiró, C. (2011). Sobre el valor educativo de los contenidos de la educación física. Tándem, 35, 68-74.

Devís, J. y Sánchez, R. (1996). La enseñanza alternativa de los juegos deportivos: antecedentes, modelos actuales de iniciación y reflexiones finales. en Moreno, J.A. y Rodríguez, P. L.: Aprendizaje deportivo (pp. 159-181). Murcia: Universidad de Murcia

Espada, M., Clemente, A.L., Santacruz, J.A. \& Gallardo, J.M. (2013). La enseñanza del deporte escolar en educación secundaria según la formación inicial del profesorado. Apunts. Educación Física y Deportes, 172, 72-81. https://doi. org/10.5672/apunts.2014-0983.es.(2013/2).112.06

Feu, S. (2002). Factores a tener en cuenta para una iniciación deportiva educativa: el contexto. Lecturas: Educación Física y Deportes, 5, 1-10.

Fraile, A. (1996). Reflexiones sobre la presencia del deporte en la escuela. Revista de Educación Física, 64, 5-10.

García, M. (1990). Aspectos sociales del deporte. Madrid: Alianza Editorial.

Gaviria, F. \& Castejón, F.J. (2013). El proceso didáctico en educación física como generador de valores y actitudes. Qualitative Research in Education, 2(2), 161-186.

Giménez, F. J. (2003). El deporte en el marco de la Educación Física. Sevilla: Wanceulen.

Giménez, F.J. \& Rodríguez, J.M. (2006). Buscando el deporte educativo, ¿Cómo formar a los maestros? Retos. Nuevas Tendencias en Educación Física, Deporte y Recreación, 9, 40-45.

Ginsburg, K. R. (2007). The importance of play in promoting healthy child development and maintaining strong parent-child bonds. Pediatrics, 179, 182-191. https://doi.org/10.1542/peds.2006-269

González, S., García, L. M., Contreras, O. \& Sánchez-Mora, D. (2009). El concepto de iniciación deportiva en la actualidad. Retos. Nuevas Tendencias en Educación Física, Deporte y Recreación, 15, 1420. https://recyt.fecyt.es/index.php/retos/article/view/34992/

Green, T. \& Gabbard, C. (1999). Do we need sportsmanship education in secondary school athletics? Physical Educator, 56(2), 98-104. https://js.sagamorepub.com/pe/article/view/2223

Gutiérrez, M. (1995). Valores sociales y deporte. Madrid: Gymnos.

Hernández, R., Fernández, C. \& Batista, P. (2006). Metodología de la investigación. México: McGraw Hill.

Jiménez, A. C. (2006). Intervención en contextos desfavorecidos a través del baloncesto. Wanceulen: Educación Física Digital, 2, 1-10.

Kemmis, S. \& Mctaggart, R. (1982). The action research planner. Vitoria: Deakin University Press.

Lee, M.J., Whitehead, J. \& Balchin, N. (2000). The measurement of values in youth sport: Development of the Youth Sport Values Questionnaire. Journal of Sport \& Exercise Psychology, 22(4), 307-326. https://doi.org/10.1123/jsep.22.4.307

Méndez, A. (1999). Modelos de enseñanza deportiva. Análisis de dos décadas de investigación. Lecturas, Educación Física y Deportes, 73.
Méndez, A. \& Fernández, J. (2011). Análisis y modificación de los juegos y deportes tradicionales para su adecuada aplicación en el ámbito educativo. Retos. Nuevas Tendencias en Educación Física, Deporte y Recreación, 19, 54-58.

Ortega, G., Giménez, F.J., Jiménez, A.C., Franco, J., Durán, L.J. \& Jiménez, P.J. (2012). Por una Educación REAL: Valores y Deporte. Iniciación al valorcesto. Madrid: Ediciones Gráficas Fundación Real Madrid.

Ortega, G., Abad, M. T., Durán, L. J., Franco, J., Giménez, F. J., Jiménez, P. J. \& Jiménez, A.C. (2015). Por una Educación REAL: Valores y Deporte. Entrenando Fútbol: Enseñando Valores. Madrid: Ediciones Gráficas Fundación Real Madrid.

Palacios, L. (2000). La entrevista. En G. Edel. Manual teóricopráctico de investigación social (pp. 99-108). Buenos Aires: Espacio editorial.

Pérez-Pueyo, A. (2016). El estilo actitudinal en educación física. Evolución en los últimos 20 años. Retos, Nuevas Tendencias en Educación Física, Deporte y Recreación, 29, 207-215. https://recyt.fecyt.es/index.php/retos/article/view/38720

Ramos, R., Valdemoros, M.A., Sanz, E. \& Ponce, A. (2007). La influencia de los profesores sobre el ocio activo de los jóvenes. Percepción de los agentes educativos más cercanos a ellos. Profesorado. Revista de Currículum y Formación del profesorado, 17(2), 1-18.

Renzi, G.M. (2009). Educación Física y su contribución al desarrollo integral de los niños en la primera infancia. Revista Iberoamericana de Educación, 50(7), 1-14.

Robles, J., Giménez, F.J. \& Abad, M.T. (2010). Motivos que llevan a los profesores de educación física a elegir los contenidos deportivos en la E.S.O., Retos, Nuevas Tendencias en Educación Física, Deporte y Recreación, 18, 5-8. https://recyt.fecyt.es/index.php/retos/article/view/34642

Silverman, S. (2005). Thinking long term: Physical education's role in movement and mobility. Quest, 57, 138-147. https://doi.org/10.1080/00336297.2005.10491847

Taylor, S. \& Bogdan, R. (1990). Introducción a los métodos cualitativos en educación. Barcelona: Paidos.

Thomas, K. T., Lee, A. M. \& Thomas, J. R. (2003). Physical education methods for elementary teachers (2nd ed., pp. 3-12). Champaign, IL: Human Kinetics.

Trepat, D. (1995). La educación en valores a través de la iniciación deportiva. En Blázquez, D. (Dir.). La iniciación deportiva y el deporte escolar (pp. 95-114). Barcelona: Inde.

Ureña, N, Alarcón, F. \& Ureña, F. (2009). La realidad de los deportes colectivos en la Enseñanza Secundaria. Cómo planifican e intervienen los profesores de Murcia. Retos. Nuevas Tendencias en Educación Física, Deporte y Recreación, 16, 9-15.

Valero, A. (2006). La iniciación al deporte del atletismo: del modelo tradicional a los nuevos enfoques metodológicos. Kronos: Revista Universitaria de la Actividad Física y el Deporte, 9, 34-44. 